\title{
Comparative Study of Retroperitoneal Laparoscopic Versus Open Ipsilateral Nephrectomy After Percutaneous Nephrostomy: A Multicenter Analysis
}

\author{
Bo Wang, MS, ${ }^{1, *}$ Ye Tian, MD, ${ }^{1, *}$ Yue Peng, MS, ${ }^{2}$ Yong Ban, MS, ${ }^{1}$ Gang Shan, MS, \\ Xianyu Tan, MS, ${ }^{3}$ Xiaohu Tang, MS, ${ }^{1}$ Guangheng Luo, MD, and Zhaolin Sun, MS ${ }^{1}$
}

\begin{abstract}
Purpose: To investigate the feasibility of retroperitoneal laparoscopic ipsilateral nephrectomy of a benign nonfunctional kidney after percutaneous nephrostomy, and to compare this method with open surgery.

Materials and Methods: Data from 70 patients who underwent simple nephrectomy from January 2014 to October 2018 at three large centers were retrospectively analyzed. All patients underwent percutaneous nephrostomy because of renal or ureteral calculi with severe hydronephrosis or pyonephrosis. Simple nephrectomy was performed via retroperitoneal laparoscopic surgery (retroperitoneal laparoscopic group; $n=33$ ) or open surgery (open group; $n=37$ ). The retroperitoneal laparoscopic and open groups were compared regarding preoperative variables (age, sex, location of surgery, hypertension, diabetes, BMI, preoperative serum creatinine level, American Society of Anesthesiologists (ASA) grade, fistula duration, fistula size, number of fistulae, and urinary tract infection), and perioperative variables (operation time, intraoperative blood loss, postoperative drainage volume, catheter indwelling time, gastrointestinal function recovery time, duration of bedrest, duration of postoperative hospitalization, postoperative hemoglobin decline, perioperative transfusion, and postoperative complications).

Results: The retroperitoneal laparoscopic group included more patients with hydronephrosis, while the open group included more patients with pyonephrosis. There were no significant differences between the two groups in age $(P=.813)$, sex $(P=.729)$, location of surgery $(P=.345)$, hypertension $(P=.271)$, diabetes $(P=.394)$, BMI $(P=.798)$, preoperative serum creatinine level $(P=.826)$, ASA grade $(P=.820)$, fistula duration $(P=.108)$, fistula size $(P=.958)$, number of fistulae $(P=.925)$, urinary tract infection $(P=.111)$, or operative time $(P=.851)$. The retroperitoneal laparoscopic group had significantly lesser intraoperative blood loss $(P=.007)$, postoperative drainage volume $(P=.008)$, shorter catheter indwelling time $(P=.002)$, gastrointestinal function recovery time $(P<.001)$, duration of bedrest $(P<.001)$, and duration of postoperative hospitalization $(P<.001)$, and lesser postoperative hemoglobin decline $(P=.035)$ compared with the open group.

Conclusions: Retroperitoneal laparoscopic ipsilateral nephrectomy is feasible for a benign nonfunctional kidney after percutaneous nephrostomy. The surgical method should be selected based on the surgeon's experience and the specific situation of the patient.
\end{abstract}

Keywords: percutaneous nephrostomy, retroperitoneal laparoscopic simple nephrectomy, open simple nephrectomy, benign nonfunctional kidneys

\footnotetext{
${ }^{1}$ Department of Urology, Guizhou Provincial People's Hospital, Guiyang, Guizhou, P.R. China.

${ }^{2}$ Department of Nephrology, The Affiliated Hospital of Guizhou Medical University, Guiyang, Guizhou, P.R. China.

${ }^{3}$ Department of Orthopedics, The Affiliated Hospital of Zunyi Medical College, Zunyi, Guizhou, P.R. China.

*These authors contributed equally to this work and should be considered co-first authors.
} 


\section{Introduction}

L APAROSCOPIC NEPHRECTOMY (LN) is indicated in the treatment of most benign renal diseases in which permanent loss of renal function has occurred. Indications for LN include nephrolithiasis, renal dysplasia, pelvi-ureteric junction obstruction, chronic pyelonephritis, tuberculosis, vesicoureteric reflux, ureterocele, polycystic kidney, and xanthogranulomatous pyelonephritis. ${ }^{1-6}$ Ipsilateral LN has been reported in patients with a history of surgical intervention. Turna et al. ${ }^{7}$ reported the feasibility of laparoscopic partial nephrectomy after previous ipsilateral renal surgery; furthermore, Aminsharifi and Goshtasbi ${ }^{8}$ reported that the difficulties associated with LN were similar for patients with previous percutaneous nephrolithotomy compared with those with previous open renal surgery. However, previous surgery can induce varying degrees of fibrosis at the surgical site or at a site away from the surgical bed. ${ }^{9}$ With the popularization of $\mathrm{LN}$ as a standard procedure at many centers, questions have arisen regarding the use of this technique in patients with a history of surgery. ${ }^{10}$

\section{Materials and Methods}

The present study was a retrospective analysis of the data from 70 patients who underwent simple nephrectomy after previous percutaneous nephrostomy from January 2014 to October 2018 at three large centers (Guizhou Provincial People's Hospital, The Affiliated Hospital of Guizhou Medical University, and The Affiliated Hospital of Zunyi Medical College). All three centers have been performing laparoscopic techniques for more than 5 years. Furthermore, every surgeon had performed more than 50 laparoscopic procedures before the beginning of the study. The present study was approved by the institutional ethics committees of all three participating centers. Simple nephrectomy was performed via the retroperitoneal laparoscopic approach in 33 patients (retroperitoneal laparoscopic group), while the other 37 underwent open surgery (open group). All patients had a unilateral benign nonfunctional kidney, with normal contralateral renal compensatory function. The causes of percutaneous nephrostomy were renal or ureteral calculi with severe hydronephrosis or pyonephrosis. All data were obtained from reviews of the medical history and physical examinations, operative and pathology reports, and anesthesia records created during hospital admission.

All patients underwent a preoperative clinical examination, including ultrasonography and renal dynamic imaging. The glomerular filtration rate of the affected kidney was $<15 \mathrm{~mL} /$ minutes, while the status of the contralateral kidney was normal. The postoperative pathology results verified that the affected kidney was a benign nonfunctional kidney in all cases. Except for percutaneous nephrostomy, patients with a history of open or laparoscopic renal ipsilateral kidney surgery were excluded from this study. The retroperitoneal laparoscopic group and the open group were compared regarding the following preoperative variables: patient age, sex, location of surgery, hypertension, diabetes, BMI, preoperative serum creatinine level, ASA grade, fistula duration ( $<1$ month versus $\geq 1$ month), fistula size, number of fistulae, and the presence of a urinary tract infection. The two groups were also compared regarding the following perioperative variables: operation time, intraoperative blood loss, postoperative drainage volume, catheter indwelling time, gastrointestinal function recovery time, duration of bedrest, duration of postoperative hospitalization, postoperative hemoglobin decline, perioperative transfusion, and postoperative complications (as assessed using the Clavien-Dindo scoring system $\left.^{11}\right)$.

\section{Results}

The baseline patient characteristics are summarized in Table 1. In the retroperitoneal laparoscopic group, percutaneous nephrostomy was performed because of renal or ureteral calculi with severe hydronephrosis in 28 of 33 patients (85\%), and because of pyonephrosis in $5(15 \%)$. The incidence of percutaneous nephrostomy because of pyonephrosis in the open group (14 of 37 patients; $38 \%$ ) was higher than that in the retroperitoneal laparoscopic group $(P=.033)$. However, the two groups did not significantly differ regarding age $(P=.813)$, sex $(P=.729)$, location of surgery $(P=.345)$, hypertension $(P=.271)$, diabetes $(P=.394)$, BMI $(P=.798)$, preoperative serum creatinine level $(P=.826)$, ASA grade $(P=.820)$, fistula duration $(P=.108)$, fistula size $(P=.958)$, number of fistulae $(P=.925)$, or urinary tract infection $(P=.111)$.

Table 1. Baseline Characteristics of the Patients

\begin{tabular}{|c|c|c|c|}
\hline Variable & $\begin{array}{l}\text { Retroperitoneal } \\
\text { laparoscopic } \\
\text { approach } \\
(\mathrm{n}=33)\end{array}$ & $\begin{array}{c}\text { Open } \\
\text { approach } \\
(\mathrm{n}=37)\end{array}$ & $\begin{array}{c}\mathrm{P} \\
\text { value }\end{array}$ \\
\hline Age (years) & $48.21 \pm 12.75$ & $49.00 \pm 14.70$ & .813 \\
\hline Gender (male) & 12 & 12 & .729 \\
\hline $\begin{array}{l}\text { Location of } \\
\text { operation (left) }\end{array}$ & 18 & 16 & .345 \\
\hline Hypertension & 11 & 8 & .271 \\
\hline Diabetes & 7 & 5 & .394 \\
\hline BMI $\left(\mathrm{kg} / \mathrm{m}^{2}\right)$ & $23.84 \pm 3.11$ & $24.44 \pm 4.60$ & .798 \\
\hline $\begin{array}{l}\text { Preoperative serum } \\
\text { creatinine level } \\
(\mathrm{mg} / \mathrm{dL})\end{array}$ & $94.5 \pm 29.58$ & $77.39 \pm 27.01$ & .826 \\
\hline \multicolumn{4}{|l|}{ ASA grade } \\
\hline $\mathrm{I}$ & 3 & 2 & .820 \\
\hline II & 27 & 32 & \\
\hline III & 3 & 3 & \\
\hline \multicolumn{4}{|l|}{ Fistula time (month) } \\
\hline$<1$ & 16 & 11 & .108 \\
\hline$\geq 1$ & 17 & 26 & \\
\hline \multicolumn{4}{|c|}{ The cause of percutaneous nephrostomy } \\
\hline $\begin{array}{l}\text { Renal or ureteral } \\
\text { calculi with } \\
\text { severe } \\
\text { hydronephrosis }\end{array}$ & 28 & 23 & .033 \\
\hline Empyema kidney & 5 & 14 & \\
\hline \multicolumn{4}{|l|}{ Size of fistula $(\mathrm{F})$} \\
\hline 14 & 10 & 11 & .958 \\
\hline 16 & 23 & 26 & \\
\hline Number of fistulas & $1.21 \pm 1.08$ & $1.18 \pm 0.93$ & .925 \\
\hline $\begin{array}{l}\text { Urinary tract } \\
\text { infection }\end{array}$ & 6 & 13 & .111 \\
\hline
\end{tabular}

ASA, American Society of Anesthesiologists; BMI, body mass index. 
The surgical outcomes of the two groups are summarized in Table 2. All of the operations were successful; however, four cases in the retroperitoneal laparoscopic group were converted to open surgery due to severe adhesion to the surrounding tissues. The two groups did not significantly differ regarding the operation time $(P=.851)$ and the incidence of perioperative transfusion $(P=.150)$. Compared with the open group, the retroperitoneal laparoscopic group had significantly lesser intraoperative blood loss $(P=.007)$, postoperative drainage volume $(P=.008)$, catheter indwelling time $(P=.002)$, gastrointestinal function recovery time $(P<.001)$, duration of bedrest $(P<.001)$, duration of postoperative hospitalization $(P<.001)$, and postoperative hemoglobin decline $(P=.035)$.

\section{Discussion}

Simple nephrectomy can be performed via the open or the laparoscopic approach. However, LN has been established as the standard of care for the management of benign nonfunctional kidneys due to its proven advantages compared with traditional open surgery, such as a good intraoperative field of view, shorter hospital stay, smaller incision, less intraoperative bleeding, and shorter recovery time. ${ }^{12}$ Laparoscopic simple nephrectomy can be performed via the transperitoneal or the retroperitoneal approach; various studies have verified that both laparoscopic approaches are feasible and have very good outcomes. ${ }^{13,14}$ The advantage of the transperitoneal approach provides a larger working space, and surgeons have access to familiar anatomical landmarks, including the liver, spleen, and colon. However, to access the kidneys, the bowel must be mobilized, increasing its risk of injury. ${ }^{15}$ The retroperitoneal approach is reliable, and enables early and direct control of the renal hilum. In contrast to transperitoneal urological surgery, the retroperitoneal laparoscopic approach negates the need to mobilize the intraperitoneal contents, thereby reducing the risk of injury. ${ }^{15,16}$ Furthermore, the retroperitoneal approach prevents injury of the abdominal organs during dissection of the kidneys, and avoids the development of postoperative adhesions and intestinal obstruction. ${ }^{17}$

Previous renal surgery is a relative contraindication for $\mathrm{LN}$, as adhesion formation increases the difficulty of the operation. ${ }^{10}$ However, with the development of laparoscopy in urology, the clinical outcomes of $\mathrm{LN}$ in patients with previous ipsilateral surgery have been investigated in retrospective studies. The retroperitoneoscopic approach reportedly reduces the incidence of complications in patients who have previously undergone open surgery. ${ }^{18}$ It has been suggested that nephrectomy or adrenalectomy should be performed via the retroperitoneoscopic approach in patients with a history of open abdominal surgery or irradiation to avoid intraperitoneal adhesions and reduce the risk of visceral injury. ${ }^{19}$ Retroperitoneoscopic surgery is also reportedly safe and feasible in patients with a localized renal tumor who have undergone previous laparotomy. ${ }^{20}$ Another study documented the feasibility of laparoscopic partial nephrectomy in patients with previous ipsilateral renal surgery. They stratified their sample $(n=25)$ into those with previous percutaneous surgery $(n=13)$ and open renal surgery $(n=12)$ and found that in both groups, the operation can be a challenge and should be limited to centers with appropriate experience in performing laparoscopic procedures. ${ }^{7}$ Percutaneous nephrostomy is a minimally invasive approach that results in less adhesion formation than open renal surgery. However, there are few reports about the role of percutaneous kidney surgery in adhesion formation, and the influence of previous percutaneous nephrostomy on the performance and outcomes of LN.

This study is the first to evaluate retroperitoneal LN of the ipsilateral kidney after percutaneous nephrostomy, and to compare this method with open surgery. There was no significant difference in operation time between the retroperitoneal laparoscopic group and the open laparoscopic group. The retroperitoneoscopic technique is associated with a significantly learning curve. ${ }^{21}$ However, the operative experience, surgeon preference, and the individual case details and anatomy certainly play roles in the selection of the surgical method. ${ }^{22}$ Although the time taken to develop retroperitoneal

Table 2. Periopreative Outcomes of Different Operation Methods

\begin{tabular}{lccc}
\hline Variable & $\begin{array}{c}\text { Retroperitoneal laparoscopic approach } \\
(\mathrm{n}=33)\end{array}$ & $\begin{array}{c}\text { Open approach } \\
(\mathrm{n}=37)\end{array}$ & $\begin{array}{c}\mathrm{P} \\
\text { value }\end{array}$ \\
\hline Operation time (minutes) & $162.24 \pm 64.81$ & $159.46 \pm 57.33$ & .851 \\
Intraoperative blood loss (mL) & $120.45 \pm 199.35$ & $362.29 \pm 468.46$ & .007 \\
Postoperative drainage volume (mL) & $150.60 \pm 133.35$ & $31.69 \pm 298.33$ & .008 \\
Catheter indwelling time (days) & $2.14 \pm 0.86$ & $3.00 \pm 1.31$ & .002 \\
Gastrointestinal function recovery time & $17.94 \pm 10.39$ & $28.70 \pm 12.69$ & $<.001$ \\
$\quad$ (hours) & $30.13 \pm 10.96$ & $49.40 \pm 14.70$ & $<.001$ \\
The leaving bed time (hours) & $3.11 \pm 1.17$ & $4.41 \pm 1.56$ & $<.001$ \\
Postoperative hospitalization time (days) & $14.32 \pm 11.87$ & $22.68 \pm 14.07$ & .035 \\
Postoperative hemoglobin decline (g/L) & 3 & 8 & .150 \\
Perioperative transfusion (mL) & 5 & 14 & .530 \\
Clavien-Dindo scoring system & 5 & 11 & .147 \\
$\quad$ Grade I & 0 & -242 \\
Grade II & - & - & - \\
Grade III & - & - & - \\
Grade IV & - & - & - \\
Grade V & 4 & - \\
Converted to open surgery & & & - \\
\hline
\end{tabular}


laparoscopy was similar in the three participating centers in the present study, it was not completely unified; with the improvement of surgical skills, the operating time of retroperitoneal laparoscopy is gradually shortened.

Postoperative complications were graded in accordance with the Clavien-Dindo scoring system, which enabled us to: (1) evaluate the safety of procedures, (2) compare different approaches based on a standardized classification system, and (3) use this grading to standardize surgical errors, and therefore improve management and prevention. ${ }^{23}$ In the retroperitoneal laparoscopic group, 5 patients experienced grade I postoperative complications, including fever $(n=4)$ and surgical site infection $(n=1)$, while no patient postoperative complications of grades II-V. However, in the open group, 11 patients developed grade I postoperative complications, including fever $(n=9)$ and surgical site infection $(n=2)$; furthermore, grade II postoperative complications developed in 3 patients, all of whom required postoperative blood transfusion. The patients with postoperative fever had urinary tract infection preoperatively.

Compared with the open group, the retroperitoneal laparoscopy group had significantly lesser intraoperative blood loss and postoperative drainage volume, and shorter catheter indwelling time, gastrointestinal function recovery time, duration of bedrest, and duration of postoperative hospitalization. Patients with renal or ureteral calculi and severe hydronephrosis have relatively mild postoperative renal and peripheral tissue adhesion after percutaneous nephrostomy. In such cases, the only marked adhesion is between the fistula and abdominal wall. Thus, the perirenal anatomy is relatively clear, and the feasibility of retroperitoneal LN is relatively high. Pyonephrosis was once considered a relative contraindication for laparoscopic surgery. ${ }^{24} \mathrm{With}$ the improvement of laparoscopy in the field of urology, retroperitoneal LN was able to achieve the same outcomes as open surgery for a tuberculous nonfunctional kidney, ${ }^{25}$ and has the advantages of less trauma, less bleeding, and more rapid postoperative recovery. ${ }^{26}$

In our experience, pyonephrosis (due to tuberculosis or pyelonephritis) is often accompanied by wide serious adhesion and fibrosis of renal tissues, disorder of the normal anatomic structure, and no obvious boundaries of the renal tissues and renal capsule. Kidney separation is very difficult, and inflammatory tissue and neovascularization can aggravate the intraoperative bleeding, and affect the operation; the renal pedicle vessel in the renal hilum is often accompanied by inflammatory or caseous tissue adhesions are difficult to separate. Furthermore, during percutaneous nephrostomy, the extravasation of the pus usually occurs, which could exacerbate the perirenal adhesions. In this study, the incidence of percutaneous nephrostomy caused by pyonephrosis in the open group was 38\%, which was greater than that in the retroperitoneal laparoscopic group. In addition, five cases of percutaneous nephrostomy were caused by pyonephrosis, and four of these cases were converted to open surgery during retroperitoneal LN. Laparoscopic separation was difficult in these four cases due to severe perirenal adhesions and fibrosis, and there was a large volume of intraoperative blood loss. Intraoperatively, we found marked adhesion between the fistula and the surrounding tissue, which also made retroperitoneal LN more difficult. Therefore, for patients with percutaneous nephrostomy caused by pyonephrosis the choice of surgical approach needs to be more cautious.
This study was a multicenter analysis. Thus, the advantages of study are that the sample size was large and the results are representative. However, there were also some limitations. This study was a retrospective analysis, and the choice of surgical method was not randomized. The open group included a greater proportion of patients with pyonephrosis, and so the results were biased, which impacts the final interpretation of the results.

\section{Conclusions}

This study demonstrated the feasibility of retroperitoneal laparoscopic ipsilateral nephrectomy of a benign nonfunctional kidney after percutaneous nephrostomy, and suggests that the choice of surgical method should be based on the experience of the surgeon and the specific situation of the patient. However, a high-quality randomized controlled trial is needed to confirm the present findings.

\section{Acknowledgments}

We thank Kelly Zammit, BVSc, from Liwen Bianji, Edanz Editing China (www.liwenbianji.cn/ac), for editing the English text of a draft of this article.

\section{Disclosure Statement}

The authors declare that they have no conflict of interest.

\section{Funding Information}

This study was funded by grants from the National Natural Science Cultivate Foundation of Guizhou Provincial People's Hospital ([2018]5764-0) and the Guizhou ScienceTechnology Support Plan Projects ([2018]2793).

\section{References}

1. Kaba M, Pirinççi N, Taken K, Geçit I, Demiray Ö, Eren H. Laparoscopic transperitoneal nephrectomy in non-functioning inflammatory kidneys with or without renal stone. Eur Rev Med Pharmacol Sci 2015;19:4457-4461.

2. Lima M, Miyaoka R, Moro J, D’Ancona C. Laparoscopic nephrectomy for xanthogranulomatous pyelonephritis: Are there predictive factors for success? Clinics 2012;67:907909.

3. Bansal RK, Kapoor A. Laparoscopic nephrectomy for massive polycystic kidney disease: Updated technique and outcomes. Can Urol Assoc J 2014;8:341.

4. Delreux A, Verhoest G, Mathieu R, Vigneau C, RiouxLeclercq N, Bensalah K. The laparoscopic nephrectomy for polycystic kidney is feasible and reproducible. Prog Urol 2013;23:394-398.

5. Verhoest G, Delreux A, Mathieu R, Patard JJ, Vigneau C, Rioux-Leclercq N, Bensalah K. Transperitoneal laparoscopic nephrectomy for autosomal dominant polycystic kidney disease. JSLS 2012;16:437.

6. Liao JC, Breda A, Schulam PG. Laparoscopic renal surgery for benign disease. Curr Urol Rep 2007;8:12-18.

7. Turna B, Aron M, Frota R, Desai MM, Kaouk J, Gill IS. Feasibility of laparoscopic partial nephrectomy after previous ipsilateral renal procedures. Urology 2008;72:584588. 
8. Aminsharifi A, Goshtasbi B. Laparoscopic simple nephrectomy after previous ipsilateral open versus percutaneous renal surgery. JSLS 2012;16:592.

9. Weibel MA, Majno G. Peritoneal adhesions and their relation to abdominal surgery: A postmortem study. Am J Surg 1973; 126:345-353.

10. Aminsharifi A, Taddayun A, Niroomand R, Hosseini MM, Afsar F, Afrasiabi MA. Laparoscopic nephrectomy for nonfunctioning kidneys is feasible after previous ipsilateral renal surgery: A prospective cohort trial. J Urol 2011;185: 930-934.

11. Mitropoulos D, Artibani W, Biyani CS, Jensen JB, Rouprêt M, Truss M. Validation of the Clavien-Dindo grading system in urology by the European Association of Urology Guidelines Ad Hoc Panel. Eur Urol Focus 2018;4: 608-613.

12. Burgess NA, Koo BC, Calvert RC, Hindmarsh A, Donaldson PJ, Rhodes M. Randomized trial of laparoscopic v open nephrectomy. J Endourol 2007;21:610 613.

13. Albala DM, Kavoussi LR, Clayman RV. Laparoscopic nephrectomy. Semin Urol 1992;10:146-151.

14. Gill IS, Clayman RV, McDougall EM. Advances in urological laparoscopy. J Urol 1995;154:1275-1294.

15. Saranchuk JW, Savage SJ. Laparoscopic radical nephrectomy: Current status. BJU Int 2005;95:21-26.

16. Novick AC. Laparoscopic radical nephrectomy: Specimen extraction. BJU Int 2005;95:32-33.

17. Garg M, Singh V, Sinha RJ, Sharma P. Prospective randomized comparison of transperitoneal vs retroperitoneal laparoscopic simple nephrectomy. Urology 2014;84:335339.

18. Seifman BD, Dunn RL, Wolf Jr JS. Transperitoneal laparoscopy into the previously operated abdomen: Effect on operative time, length of stay and complications. J Urol 2003;169:36-40.

19. Viterbo R, Greenberg RE, Al-Saleem T, Uzzo RG. Prior abdominal surgery and radiation do not complicate the retroperitoneoscopic approach to the kidney or adrenal gland. J Urol 2005;174:446-450.

20. Mita K, Shigeta M, Mutaguchi K, Matsubara A, Yoshino T, Seki M, Yasumot H. Urological retroperitoneoscopic sur- gery for patients with prior intra-abdominal surgery. Eur Urol 2005;48:97-101.

21. Rassweiler JJ, Seemann O, Frede T, Henkel TO, Alken P. Retroperitoneoscopy: Experience with 200 cases. J Urol 1998;160:1265-1269.

22. Challacombe B, Sahai A, Murphy D, Dasgupta P. Laparoscopic retroperitoneal nephrectomy for giant hydronephrosis: When simple nephrectomy isn't simple. J Endourol 2007;21:437-440.

23. Rassweiler JJ, Rassweiler MC, Michel MS. Classification of complications: Is the Clavien-Dindo classification the gold standard? EurUrol 2012;62:256-258.

24. Simon SD, Castle EP, Ferrigni RG, Lamm DL, Swanson SK, Novicki DE, Andrews PE. Complications of laparoscopic nephrectomy: The Mayo clinic experience. J Urol 2004;171:1447-1450.

25. Hemal AK, Gupta NP, Kumar R. Comparison of retroperitoneoscopic nephrectomy with open surgery for tuberculous nonfunctioning kidneys. J Urol 2000;164:32-35.

26. Hemal AK, Gupta NP, Wadhwa SN, Goel A, Kumar R. Retroperitoneoscopic nephrectomy and nephroureterectomy for benign nonfunctioning kidneys: A single-center experience. Urology 2001;57:644-649.

Address correspondence to:

Guangheng Luo, MD

Department of Urology

Guizhou Provincial People's Hospital

Guiyang

Guizhou 550002

P.R. China

E-mail: luoguangheng1975@163.com

Zhaolin Sun, MS

Department of Urology

Guizhou Provincial People's Hospital

Guiyang

Guizhou 550002

P.R. China

E-mail: sz15926186@163.com 